\title{
NUMERICAL INVESTIGATION OF AN UNSTEADY INJECTION ADAPTED TO THE CONTINUOUS DETONATION WAVE ROCKET ENGINE OPERATION
}

\section{T. Gaillard, D. Davidenko, and F. Dupoirieux}

ONERA

Chemin de la Hunière, BP 80100, Palaiseau CEDEX 91123, France

\begin{abstract}
Detonation applied to propulsion could result in a promising increase of the thermodynamic efficiency of the engine cycle. Numerical simulations of the detonation propagating in the Continuous Detonation Wave Rocket Engine (CDWRE) are currently performed but still do not account for realistic injection process. The assumption of an ideal injected premix is generally chosen for convenience to obtain theoretical results. Comparison of the numerical results with experiments is difficult because of the clear difference of the injection configurations. Some physical aspects of the separate injection of the components used in experiments are not clearly assessed. This study is included in a wider numerical project aimed at designing and optimizing a realistic CDWRE. The optimization process is presently focused on the injector. One element of the injection hole pattern is considered assuming that this element is periodically repeated over the injector head. The aim of the work presented here is to model and analyze the refill process of the components in the combustion chamber behind the rotating detonation. The simulation starts just after the passage of the detonation over the considered injection element. This simulation gives information on the way the injected propellants recreate the reactive mixture for the next detonation. In the first step, two-dimensional (2D) computations helped us to set up the methodology and to study the dynamic response of the fresh components injected. A comparison between $2 \mathrm{D}$ homogeneous and separate injections is provided. In the second step, three-dimensional (3D) computations have been performed with a separate injection suitable for the CDWRE operation. Some performance parameters are evaluated such as mixing efficiency or filling of the domain.
\end{abstract}

(C) The Authors, published by EDP Sciences. This is an open access article distributed under the terms of the Creative Commons Attribution License 4.0 (http://creativecommons.org/licenses/by/4.0/). 


\section{INTRODUCTION}

It is widely accepted that detonation engines represent an alternative to the conventional ones, whether it is applied to aircraft or rocket propulsion. Its beneficial effect on the thermodynamic cycle has been theoretically proved by Zel'dovich [1] and Wintenberger and Shepherd [2]. The additional pressure gain provided by detonation makes it a more interesting combustion regime than classical deflagration.

Over the past decade, Rotating Detonation Engines (RDE) have aroused scientists' interest in both numerical and experimental fields. Since the first numerical simulations of a continuous detonation under 2D hypothesis [3], some important achievements have greatly helped to simulate RDE operation in three dimensions. Eude et al. [4] have succeeded in computing a 3D rotating detonation in an RDE chamber with a uniformly distributed injection using adaptive mesh refinement. Three-dimensional propagation of a periodic rotating detonation has been studied by Schwer and Kailasanath [5] over a row of several holes for a premix injection. They have observed the feedback pressure effect on the continuous injection and the consequences on detonation stability. Recently, Stoddard and Gutmark [6] have tried to simulate a 3D rotating detonation in a centerbodiless RDE fed with a separate injection. Only one detonation has been propagated in the chamber, indicating the need of additional improvements.

This numerical study focuses on analyzing an injector applicable to a rocket engine operating in the continuous detonation mode, which is also called CDWRE. In the CDWRE combustor, stable propagation of detonation waves is ensured by a layer of fresh mixture continuously formed close to the injector head. The present authors consider that detonation waves travel along the azimuth of an annular combustor. The feeding process involves gaseous injection of two propellants, $\mathrm{H}_{2}$ and $\mathrm{O}_{2}$, at a stoichiometric ratio. As detonation speed exceeds $2 \mathrm{~km} / \mathrm{s}$, the refill process is a critical phase of the CDWRE operation and must not exceed a few tens of microseconds. In this short amount of time, high uniformity of the fresh mixture is required as well as low losses of the injection total pressure to keep the theoretical benefit.

The propellants can be introduced separately or as a premix in the combustor. Premixed injection represents the ideal injection case for which the highest possible compression from the detonation can be obtained. Although it is massively used to simplify the computation of rotating detonation, it is hardly applicable to experiments. Detonation is likely to propagate back into the feeding system through the injection holes if their diameter is not inferior to a critical tube diameter dependent on the initial pressure of the fresh mixture [7]. Some experimental attempts have been made by Andrus et al. [8] to propagate two consecutive detonations in a premixed layer refilled as if it were in a RDE. The appearance of flashbacks and blowoffs confirms that it is not a safe operation to use a premix. 
In case of transmission into the feeding collector and mixing device, a brutal energy release in a confined volume can cause an important deterioration of the combustor, as reported by Thomas et al. [9]. To avoid the aforementioned risk, separate injection is commonly used in experiments. The advantage of the perfect mixing is now lost. Thus, the issue is to obtain fast and efficient mixing with turbulent structures while limiting the total pressure losses to preserve the potential of detonation. Some experimental studies tend to show that mixing is essential for detonation stability but still little information is available to characterize mixing state in the fresh layer. What is known is that a deficit of $10 \%$ to $40 \%$ of the detonation speed from the theoretical Chapman-Jouguet (CJ) velocity is generally observed $[10,11]$. This difference may be due to inhomogeneities of composition and pressure losses. That is why, this numerical study is dedicated to the characterization and optimization of separate injection. Recent numerical works have been undertaken to propose new configurations for mixing propellants. Stoddard et al. $[12,13]$ tried different geometries for separate air and hydrogen injection tubes with a single detonation propagation over a linear series of 10 injection elements. They wanted to study the expansion of burnt gases and the ability to refill the fresh mixture by propagating a real detonation. Such kind of simulation must be developed to find out more about unsteady process occurring during RDE operation.

The present study is included in a wider project aimed at computing an entire CDWRE chamber with a suitable feeding system. Besides mixing issues, we are also interested in evaluating the propulsive performance of a CDWRE. Future studies may involve full-scale engine design with a thrust nozzle. A specific modeling approach for the injector might be needed to have such a simulation at affordable cost. In a previous analysis [14], 3D Large Eddy Simulation (LES) was performed to compute the mixing of propellant jets and to evaluate $\mathrm{H}_{2} / \mathrm{O}_{2}$ mixing efficiency and pressure losses after a sufficient time to establish the flow. Since then, we are progressing step by step to understand the mixing interactions at low scales and to choose the best way to inject the components. The focus is on understanding the mixing interactions at the scale of the injected jets. The turbulence integral scale is considered to be of the order of the tube diameters. Then, a single element simulation on a fine mesh enables us to obtain a resolved scale about one tenth of the integral scale. Even if the simulated conditions did not correspond to the CDWRE chamber environment, it was necessary to choose the injection element design for the following study. This paper presents the latest new simulations in which unsteady refilling process after detonation passage is taken into account. Even if the injection blocking and jet development phases are reproduced, chemical reactions in the injected propellants due to partial mixing with the burnt products are not modeled. Detonation propagation is not directly simulated but the expansion of burnt gases past the detonation wave is modeled. This new methodology is presented hereafter and together with results for premixed and separate injection in $2 \mathrm{D}$ and $3 \mathrm{D}$ simulations. 


\section{MODELING STRATEGY FOR THE CONTINUOUS DETONATION WAVE ROCKET ENGINE INJECTOR}

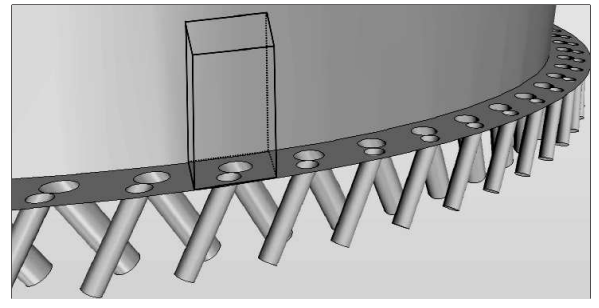

Figure 1 Example of one ring of the hole pattern repeated periodically along the chamber azimuth. The wireframe outlines the domain extracted for the computational study of a single injection element

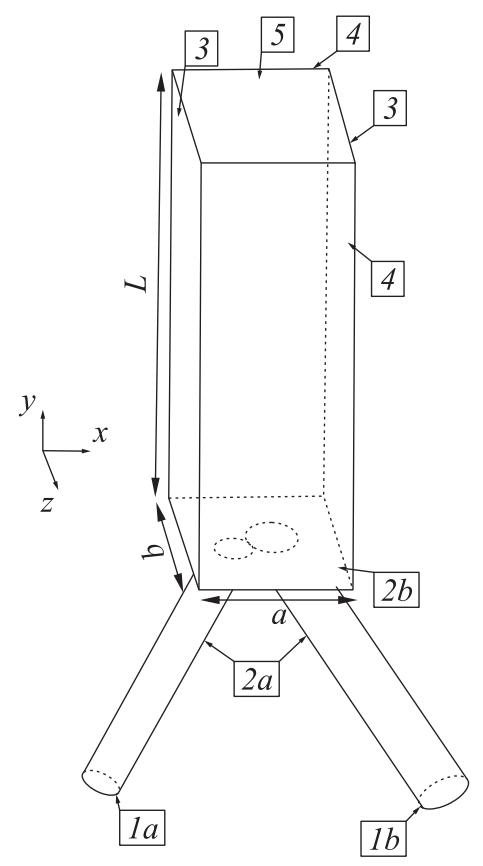

Figure 2 Boundaries of the reduced computational domain for the injection study
Before computing a complete CDWRE chamber, it is useful to focus on the propellant injection. Two points are of particular interest when designing and analyzing an injection process: $(i)$ the way reactants mix; and (ii) the total pressure losses involved. After optimization, the injection device will be studied under conditions of the $3 \mathrm{D}$ propagation of a rotating detonation.

The present study is based on a particular injector design. It is assumed that the CDWRE has an annular cylindrical combustor delimited by inner and outer walls and the injector face. The injector is designed as a repetition of one element both along the azimuth and radius. An example of such a geometry is depicted in Fig. 1.

From the whole injector, one single element is extracted (see Fig. 1) and becomes the reduced computational mixing domain shown in Fig. 2. Flow interactions between the adjacent elements can be taken into account with either periodic or symmetric boundary conditions ( 3 and 4 ) according to the adopted repetition principle. In this study, 3 and 4 are the periodic boundaries. The inlet conditions ( $1 \mathrm{a}$ and $1 \mathrm{~b}$ ) can be defined by imposing a mass flow rate or a total pressure together with a total temperature and injected gas 


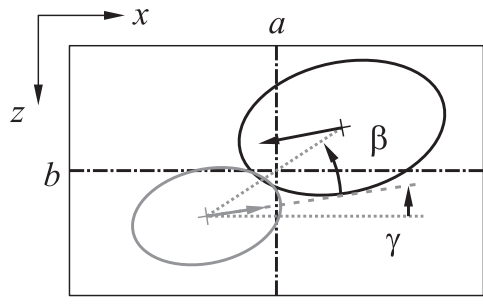

(a)

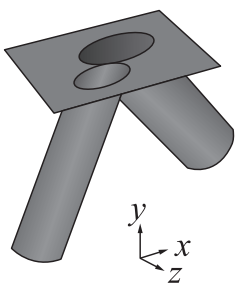

(b)

Figure 3 Injection element design: $(a)$ injection holes on the injector face $(a$ $=3.45 \mathrm{~mm}, b=3.41 \mathrm{~mm}, \beta=45^{\circ}, \gamma=13^{\circ}, d_{\mathrm{H}_{2}}=1 \mathrm{~mm}$, and $\left.d_{\mathrm{O}_{2}}=1.41 \mathrm{~mm}\right)$. Hydrogen tube is in grey and oxygen is in black; and (b) $3 \mathrm{D}$ configuration of the semi-impingement injection element

composition. For the outlet 5, only pressure is prescribed. The boundary layer is not taken into account. Hence, the mesh is not refined close to the tube walls and the injection plane. As a consequence, slip conditions are applied on tube walls $(2 \mathrm{a}$ and $2 b)$. This condition does not feature reality but helps to better respect the mass flow rates of each propellant that leads to stoichiometric proportions, which is more convenient for the analysis.

Geometric design for the injection element is presented in Fig. 3 with all the parameters of interest as defined for the best configuration in the previous study (referred to as $3 \mathrm{a}^{\prime}$ ) [14]. This configuration is a semi-impingement of propellant jets with suitable distribution. It is our $3 \mathrm{D}$ case for this study.

\section{NUMERICAL PROCEDURES}

Computations are done with the CEDRE code $[15,16]$, which is a multiphysics software developed at ONERA to solve energetic and propulsion related problems. Only the gaseous solver CHARME has been used to perform the simulations.

The compressible Navier-Stokes equations are solved for a nonreacting flow on a regular mesh with a 50-micron cell size in order to capture a wide range of vortex scales. As the mixing process is strongly unsteady due to hydrodynamic instabilities, the LES approach is chosen to simulate the energetic turbulent scales. The subgrid viscosity is modeled by the Smagorinsky model for its simplicity and as a first hypothesis to account for the nonresolved turbulent scales. A finite-volume method for general unstructured meshes is adopted for the spatial discretization. Second-order accuracy is obtained with a MUSCL (Monotonic Upstream Scheme for Conservation Laws) interpolation scheme coupled with the 
Van Leer slope limiter for the convective fluxes. The viscous fluxes are computed using an adapted second-order centered scheme. An implicit time integration is performed based on the Generalized Minimal RESidual (GMRES) method to solve the linearized equations system.

Hereafter, two different numerical procedures are proposed to study, on the one hand, an established flow with prescribed Mach injection and outlet pressure; and on the other hand, a transitory phase corresponding to the refill of the chamber linked to the expansion of the burnt gases produced after detonation passage over the injection element. While the first procedure has already been developed [14], the second one is explained and tested first on 2D cases and then applied to the $3 \mathrm{D}$ configuration.

\subsection{Particular Procedure for Established Injection Regimes}

In [14], three different jet interactions (sheared, impingement and semiimpingement) with two arrangements (periodic or symmetric repetition) along the $x$-axis were compared. The aim of this first analysis was to determine the best injection element based on mixing efficiency and total pressure recovery after a sufficient time to establish the flow. The present authors selected the semiimpingement configuration with periodic arrangement and this selected configuration is used for the calculations presented in sections 4 and 5. A mass flow rate is imposed at the inlets in order to obtain a stoichiometric composition with the following fluxes related to the injection sections of each component: $j_{m, \mathrm{O}_{2}}=844 \mathrm{~kg} /\left(\mathrm{m}^{2} \mathrm{~s}\right)$ and $j_{m, \mathrm{H}_{2}}=213.5 \mathrm{~kg} /\left(\mathrm{m}^{2} \mathrm{~s}\right)$. The corresponding injection Mach is equal to 0.8 with a prescribed pressure of $0.25 \mathrm{MPa}$ at the initial time and on the outlet boundary 5 (see Fig. 2). Total temperature is set to $300 \mathrm{~K}$. Tube lengths are set to $4 d_{\mathrm{H}_{2}}$. Mixing domain length $L$ is equal to $15 d_{\mathrm{H}_{2}}$. A first computation is made with a large time step of $1 \mu$ s with a first-order implicit Euler method to evacuate the initial conditions for a physical time of $1 \mathrm{~ms}$. Then, a second-order implicit Runge-Kutta method is applied to simulate 3 to 4 residence times with a 0.01 -microsecond time step. The flowfield of interest is obtained with another 0.01-microsecond time step computation during a physical time of $100 \mu \mathrm{s}$.

\subsection{Particular Procedure for Transitory Injection Regimes}

\subsubsection{Definition of the initial conditions}

The initial conditions are the key point of the modeling methodology. The transitory refill of the domain must be representative of what is likely to happen 


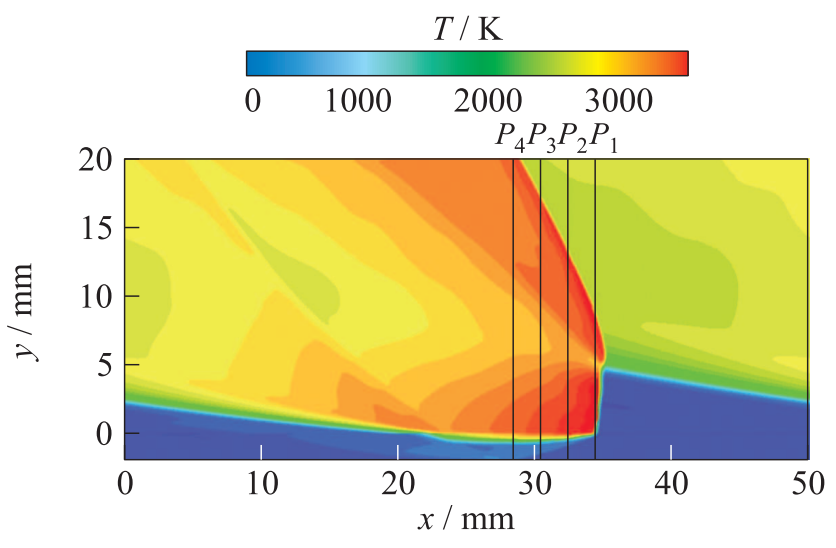

Figure 4 Results from a 2D CDWRE case: instantaneous temperature field with four lines for pressure profile extraction

from the very beginning of the process. Before starting studies on the reactant mixing in the reduced domain, $2 \mathrm{D}$ computations of a rotating detonation were performed with the CEDRE code to observe the physical and representative content of the unsteady refill. Stoichiometric $\mathrm{H}_{2} / \mathrm{O}_{2}$ premix was injected through the lower boundary of a rectangular periodic domain with a mass flux of $100 \mathrm{~kg} /\left(\mathrm{m}^{2} \mathrm{~s}\right)$ in the chamber and an established flowfield was obtained with a detonation speed $D=2670 \mathrm{~m} / \mathrm{s}$. An instantaneous temperature field from this simulation is presented in Fig. 4. The feeding slot corresponds to the area of negative $y$-value where chemical reactions are not activated. From the slot to the chamber, the depth of the domain cross section is increased by a coefficient $10 / 3$. As the detonation pressure at $y=0$ is stronger than the injection pressure, a shockwave propagates in the feeding slot but it does not affect the main physical phenomena. The higher burnt gases pressure leads to the stop of the injection for a few microseconds.

The evolution of the vertical Mach number $\mathrm{M}_{y}$ and the hydrogen mass fraction $Y_{\mathrm{H}_{2}}$ are extracted along the level $y=0.5 \mathrm{~mm}$ and shown in Fig. 5. The coordinate $x$ in Fig. 4 has been transformed in the time variable in Fig. 5 according to:

$$
t=\frac{\left(x_{\text {front }}-x\right)+0.05 \min \left[\left(x_{\text {front }}-x\right), 0\right] /\left(x_{\text {front }}-x\right)}{D}
$$

where $x_{\text {front }}$ is about $0.034 \mathrm{~m}$. In Fig. 5, one can see that it takes $6 \mu$ s to reinject the right $Y_{\mathrm{H}_{2}}$ value. This blocking time represents up to $30 \%$ of the refill period, the later evaluated to $18.7 \mu \mathrm{s}$. When it is refilled, the Mach number of the fresh mixture continuously increases to reach a value higher than 1 . This transition leads to some pressure losses in the propellants flow before detonation 


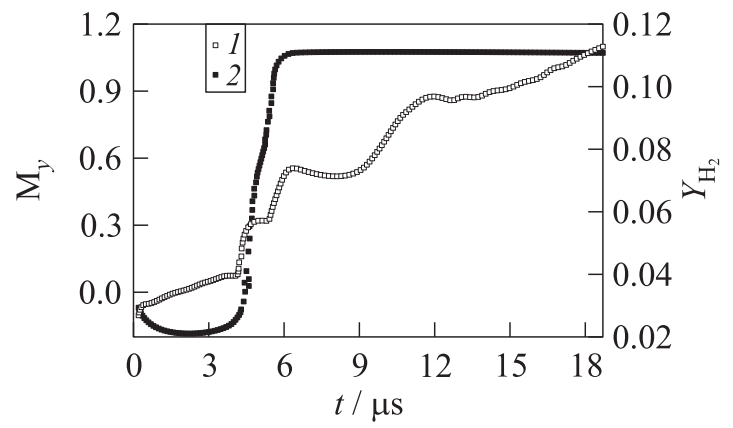

Figure 5 Results from a 2D CDWRE case: vertical Mach number (1) and hydrogen mass fraction (2) profiles at the injection plane $y=0.5 \mathrm{~mm}$ during a refill period

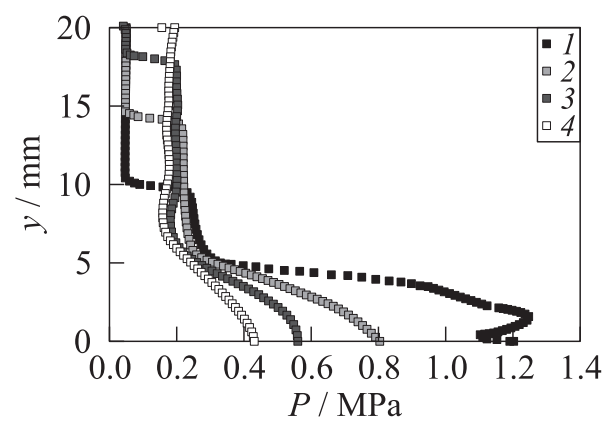

Figure 6 Pressure profiles behind the detonation extracted along the vertical lines in Fig. 4: $1-P_{1}$; $2-P_{2} ; 3-P_{3} ;$ and $4-P_{4}$

These profiles account for pressure evoaforementioned calculation (Fig. 6). These profiles account for pressure evolution across the wave structure composed of the rarefaction waves, the slip line and the shockwave. Only the rarefaction wave is important for the injection as the others never reach the injection plane.

With our methodology, we want to produce initial conditions and control the expansion process of the burnt gases that are local in $x$ but sufficiently representative for our mixing study. The wave structures displayed in Fig. 6 resemble to the solutions of a Riemann problem extracted at particular times in an $(y-t)$ diagram. To model this Riemann problem, let us first assume that the detonation propagates with a plane front whose height is $h$ (Fig. 7). The initial discontinuity occurs at the top of the detonation front at $y\left(t_{0}\right)=h$. The discontinuity divides 
the $t_{0}$ state in two homogeneous states, referred to as the right "rh" $(y<h)$ and left "lh" $(y>h)$ states. For convenience, the $(y-t)$ diagram is displayed with a representation adapted to the detonation front. Also, the $t$-axis is turned into a $x$-axis to be related to Fig. 4 .

The $l h$ state is defined by the CJ conditions, representing the burnt gases state just behind the detonation front. It is calculated by extracting the mean properties of the fresh gases $(m)$ just in front of the detonation wave from the CDWRE case (see Fig. 4), which are the following:

$$
\begin{gathered}
P_{m}=0.062 \mathrm{MPa} ; \quad T_{m}=239.7 \mathrm{~K} ; \quad \rho_{m}=0.377 \mathrm{~kg} / \mathrm{m}^{3} ; \\
Y_{\mathrm{H}_{2}, m}=0.1111 ; \quad Y_{\mathrm{O}_{2}, m}=0.8889
\end{gathered}
$$

where $P$ is the pressure; $T$ is the temperature; $\rho$ is the density; and $Y$ is the mass fraction. The corresponding CJ conditions have been evaluated:

$$
\begin{array}{rlrl}
P_{\mathrm{CJ}} & =1.45 \mathrm{MPa} ; T_{\mathrm{CJ}}=3620 \mathrm{~K} ; \quad \rho_{\mathrm{CJ}}=0.698 \mathrm{~kg} / \mathrm{m}^{3} ; \\
Y_{\mathrm{H}, \mathrm{CJ}} & =0.00574 ; \quad Y_{\mathrm{H}_{2}, \mathrm{CJ}}=0.0226 ; & Y_{\mathrm{H}_{2} \mathrm{O}, \mathrm{CJ}}=0.664 ; \\
Y_{\mathrm{O}, \mathrm{CJ}} & =0.0431 ; \quad Y_{\mathrm{O}_{2}, \mathrm{CJ}}=0.108 ; & Y_{\mathrm{OH}, \mathrm{CJ}}=0.15656 .
\end{array}
$$

The $r h$ state defines some artificial state to control the expansion process of burnt gases. To obtain this state, it is assumed that the CJ burnt gases from the previous detonation have undergone an isentropic expansion, from the CJ state with vertical Mach $\left(\mathrm{M}_{\mathrm{CJ}}\right)$ equal to 0 to a prescribed vertical Mach $\left(\mathrm{M}_{r h}\right)$ in the $r h$ state. The mass fractions are constant across the discontinuity since in the present study, chemical reactions have been neglected.

The value of $\mathrm{M}_{r h}$ influences on the opening of the rarefaction wave. For example, for a supersonic $\mathrm{M}_{r h}$, the area that influences the injection plane is

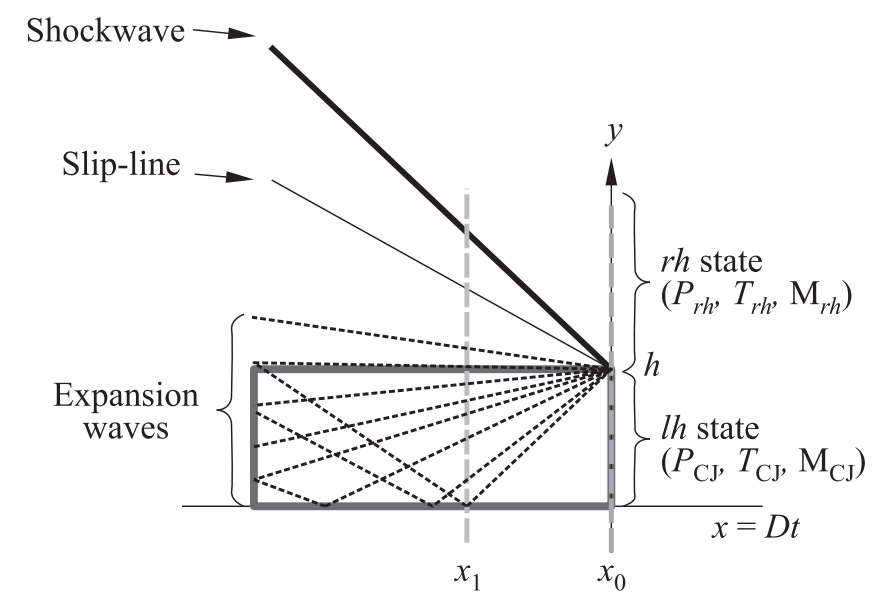

Figure 7 Modeling of the detonation front by a Riemann problem 


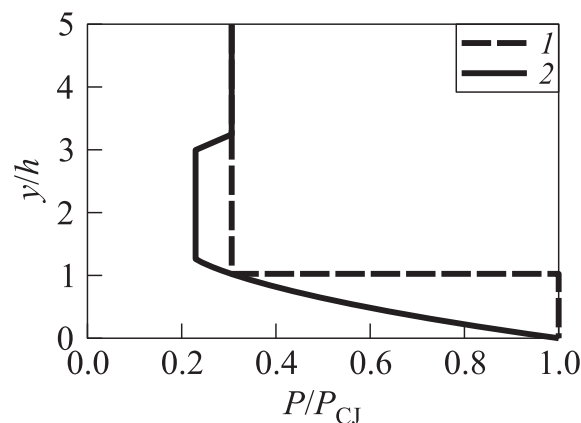

(a)

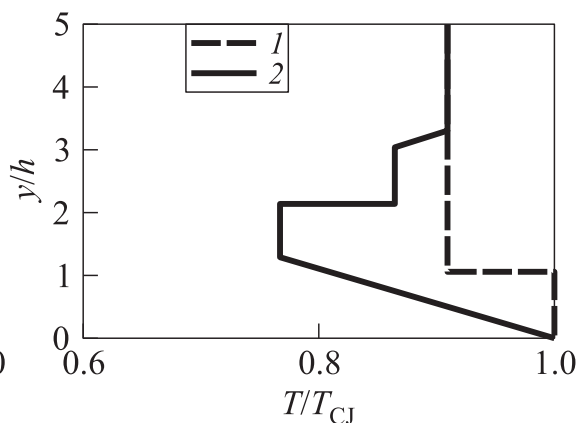

(b)

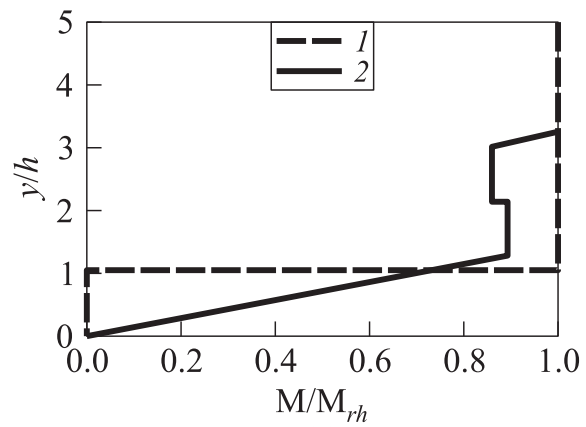

(c)

Figure 8 Initial conditions derived from the Riemann problem solution at $x_{0}(1)$ and $x_{1}(2)$ according to Fig. 7: $(a)$ pressure; (b) temperature; and (c) vertical Mach number

delimited by the rectangle in Fig. 7. Indeed, the sonic Mach limit is located at $y=h$. All the waves above this limit will not reach the injection plane.

From the initial discontinuity between the CJ and the $r h$ states, the exact Riemann problem has been solved with an in-house code. The self-similar solution can be obtained from $t_{0}\left(x_{0}\right)$ to $t_{1}\left(x_{1}\right)$ where $t_{1}$ is the instant at which the expansion wave reaches the injection plane. After this time, the solution is not considered because the reflection of the expansion fan is out of the scope of the Riemann problem. We obtain a multitude of possible profiles between $x_{0}$ and $x_{1}$ to be tested as initial conditions. The solutions obtained in $x=x_{0}$ and $x=x_{1}$ are given in Fig. 8 for $\mathrm{M}_{r h}=1.5$.

Note that with a high vertical velocity in the chamber, the expansion process leads to an expansion pressure that is lower than $P_{r h}$. Hence, the evolution of $P, T$, and $\mathrm{M}$ is reversed from $y / h=3$ to 3.5 , compared to what the profiles in Fig. 6 suggest. Nevertheless, with the proposed technique, a suitable $P(t)$ evolution of the expansion process has been obtained along $y$ in the zone of influence $(y<h)$. 
Now, let us make the following assumptions to apply these profiles as initial conditions for the injection and mixing simulation:

(1) the injection element is sufficiently small to neglect flow parameter variation along $x$ and $z$; and

(2) $x$ - and $z$-wise velocities are initially set to 0 . By analyzing numerical results, it was found that the $x$-wise velocity is important right behind the detonation but then it rapidly decreases and is relatively low during the reinjection phase.

\subsubsection{Two-dimensional test cases}

For the first time, the methodology is tested on simple $2 \mathrm{D}$ cases for premixed and separate injection. The corresponding $2 \mathrm{D}$ domains are presented in Fig. 9. For the separate injection, the widths of the $\mathrm{H}_{2}$ and $\mathrm{O}_{2}$ injection pipes are equal to $d / 3$ and $2 d / 3$, respectively, with $d=1 \mathrm{~mm}$. The mesh is refined in area 1 in Fig. 9 $(-15 d<y<15 d)$ to obtain cells of 50 -micron side, whereas it is coarsened in areas 2. The upper initial conditions $(y>0)$ are determined by the present authors' previous methodology whereas the lower initial conditions $(y<0)$ correspond to a uniform flow separated by a discontinuity at $y=0$. By doing this, it is assumed that before the detonation arrival, the injection was completely established; then, the fresh mixture above the injection plane was instantly burnt by detonation without perturbing the initial state in the pipes.

Two validation cases, referred to as 1.1 and 1.2, are introduced in Table 1 taking the solutions of the Riemann problem (see Fig. 8) in $x=x_{0}$
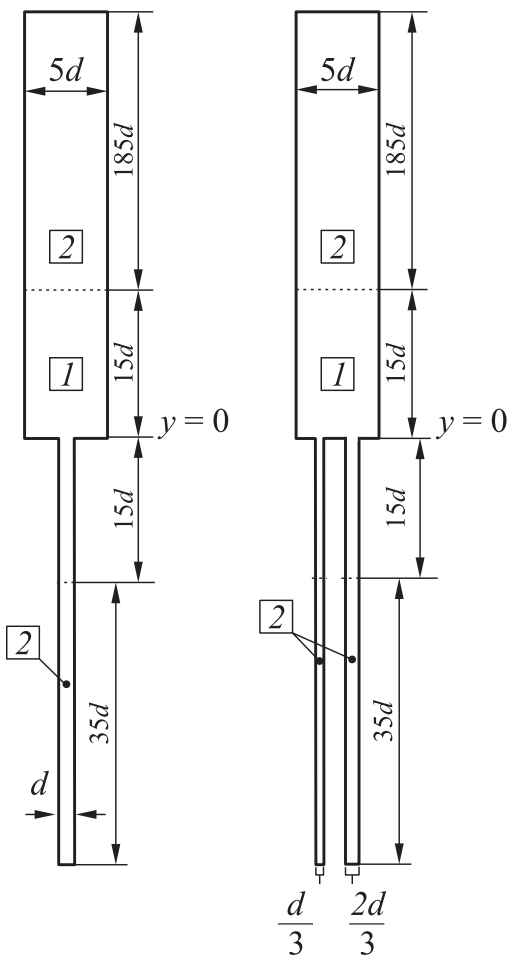

(a)

(b)

Figure 9 Domains for 2D computations: $(a)$ premixed injection; and $(b)$ separate injection and $x=x_{1}$ as the initial upper conditions, with $\mathrm{M}_{r h}=1.5$. They correspond to premixed injection with an initial Mach $\mathrm{M}_{\mathrm{ini}}=1$ in the injection pipes. 
Table 1 Two-dimensional injection cases for the validation of the initial conditions

\begin{tabular}{ccccc}
\hline Case & Premix/separate & $\mathrm{M}_{r h}$ & $\mathrm{M}_{\mathrm{ini}}$ & Initial condition for $y>0$ \\
\hline 1.1 & \multirow{2}{*}{ premix } & \multirow{2}{*}{1.5} & \multirow{2}{*}{1.0} & $x_{0}$ \\
1.2 & & & & $x_{1}$ \\
\hline
\end{tabular}

The inlet conditions set at $y=-50 d$ for premixed injection are defined by total pressure $\left(P_{t, \text { inj }}\right)$ and temperature $\left(T_{t, \text { inj }}\right)$. From a given mass flux $j_{m}$ $=100 \mathrm{~kg} /\left(\mathrm{m}^{2} \mathrm{~s}\right)$ related to the chamber cross section, the total injection pressure was derived through the following relationship:

$$
\begin{aligned}
P_{t}=P_{s}\left(1+\frac{\gamma-1}{2} \mathrm{M}_{\mathrm{ini}}^{2}\right)^{\gamma /(\gamma-1)} & \\
= & \frac{j_{m} \sqrt{2 R T_{i}}}{A_{\% \text { inj }} \sqrt{W \gamma(\gamma+1)}} \frac{1}{\mathrm{M}_{\text {ini }}}\left(1+\frac{\gamma-1}{2} \mathrm{M}_{\text {ini }}^{2}\right)^{\gamma /(\gamma-1)}
\end{aligned}
$$

where $W$ is the gas molar mass in $\mathrm{kg} / \mathrm{mol} ; A_{\% \text {,inj }}$ is the injection area ratio equal to 0.2 ; and $R=8.314 \mathrm{~J} / \mathrm{mol} / \mathrm{K}$ and $\gamma=1.4$ are the fresh mixture parameters. The initial static conditions in the tubes $\left(P_{s, \text { ini }}\right.$ and $\left.T_{s, \text { ini }}\right)$ are calculated to comply with the initial injection Mach number $M_{\text {ini }}=1$ :

$$
\begin{aligned}
& P_{t, \text { inj }}=0.333 \mathrm{MPa} ; \quad T_{t, \text { inj }}=300 \mathrm{~K} ; \\
& P_{s, \text { ini }}=0.176 \mathrm{MPa} ; \quad T_{s, \text { ini }}=250 \mathrm{~K} ; \quad v_{\text {ini }}=492 \mathrm{~m} / \mathrm{s} .
\end{aligned}
$$

In Fig. 10, pressure and temperature evolutions at the injector exit obtained for cases 1.1 and 1.2 are compared to the aforementioned CDWRE calculation.

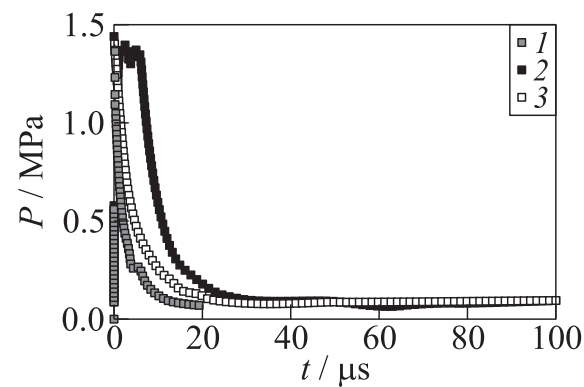

(a)

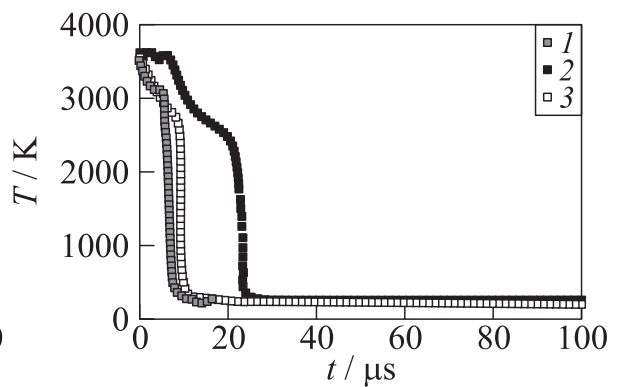

(b)

Figure 10 Comparison of the pressure $(a)$ and temperature $(b)$ evolution at the injector exit for the CDWRE (1) and cases 1.1 (2) and 1.2 (3) 
Table 2 Two-dimensional premixed injection cases for studying the effect of $\mathrm{M}_{r h}$

\begin{tabular}{ccc}
\hline Case & $\mathrm{M}_{r h}$ & $\mathrm{M}_{\mathrm{ini}}$ \\
\hline 2.1 & 0.8 & \\
2.2 & 1.0 & \\
2.3 & 1.5 & 0.8 \\
2.4 & 2.0 & \\
\hline
\end{tabular}

Table 3 Comparison of the initial $\left(\mathrm{M}_{\mathrm{ini}}\right)$ and stabilized injection Mach $\left(\mathrm{M}_{\mathrm{inj}}\right)$ after burnt gases expansion

\begin{tabular}{ccccc}
\hline Case & $\mathrm{M}_{r h}$ & $P_{\mathrm{ch}}, \mathrm{MPa}$ & $\mathrm{M}_{\mathrm{ini}}$ & $\mathrm{M}_{\mathrm{inj}}$ \\
\hline 2.1 & 0.8 & 0.4 & & 0.3 \\
2.2 & 1.0 & 0.25 & 0.8 & 0.7 \\
2.3 & 1.5 & $0.045-0.04$ & & sonic \\
2.4 & 2.0 & $0.03-0.025$ & & sonic \\
\hline
\end{tabular}

For the CDWRE case, the profiles $P(x)$ and $T(x)$ are extracted at $y=0.5 \mathrm{~mm}$ and are then plotted vs. time $t$ according to Eq. (1). For cases 1.1 and 1.2, the evolutions of $P$ and $T$ are given at the same $y$ coordinate as in the CDWRE case.

The physical effect of the burnt gases expansion is better featured in case 1.2. Despite a slower pressure decrease, it can be considered that the time evolution obtained with the conditions of case 1.2 correctly reproduces the evolution given by the CDWRE calculation. The time at which low-temperature propellants replace the burnt gases is quite satisfactory in case 1.2 , whereas this time is much too long in case 1.1. Therefore, the initial conditions deduced from the solution of the Riemann problem at $x=x_{1}$ are considered appropriate for the following calculations.

Now, let us analyze the effect of $\mathrm{M}_{r h}$ on the refill process. Cases 2.1 to 2.4 (Table 2) are the premix injection cases with subsonic $\mathrm{M}_{\mathrm{ini}}$ and $\mathrm{M}_{r h}$ varying from 0.8 to 2 .

As expected for cases 2.1 to 2.4, the stabilized injection Mach $\left(\mathrm{M}_{\mathrm{inj}}\right)$ after burnt gases expansion during $100 \mu$ s is different from the initial prescribed $\mathrm{M}_{\mathrm{ini}}$ because the final pressure level $\left(P_{\mathrm{ch}}\right)$ in the chamber is conditioned by $\mathrm{M}_{r h}$. These dependences are shown in Table 3. With $M_{r h}$ greater than 1.5 (cases 2.3 and 2.4), the acceleration induced by the burnt gases movement in the chamber produces a transition from the subsonic injection regime (Mach of 0.8) to a sonic injection regime. Conversely, $\mathrm{M}_{r h}=0.8$ (case 2.1) restrains the refill process to $\mathrm{M}_{\mathrm{inj}}=0.3$.

The actual injected mass flow rate in cases 2.1 to 2.4 is then different from the initial value since the injected flow adapts to the stabilized chamber conditions. The nondimensional mass of the fresh mixture $m_{\%}$ injected in the chamber is evaluated as follows:

$$
m_{\%}=\frac{\int_{S_{\mathrm{ch}}} \rho\left(Y_{\mathrm{H}_{2}}+Y_{\mathrm{O}_{2}}\right) d S}{\rho_{m} S_{\mathrm{det}}}
$$

where $S_{\text {det }}=5 d h$ is the imposed detonation front area; $S_{\mathrm{ch}}$ is the domain area above the injection plane; and $\rho_{m}$ is the mean density of the fresh mixture 


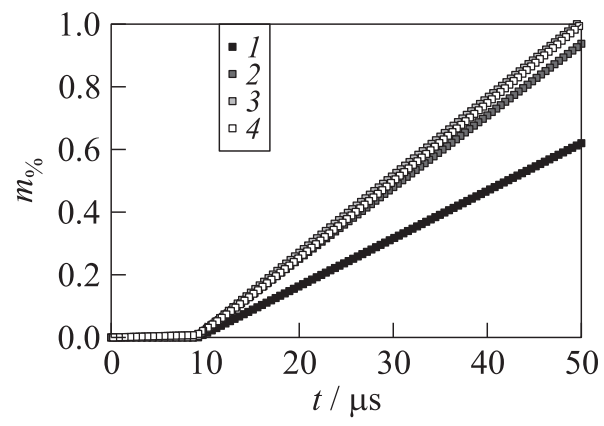

Figure 11 Fraction of the initial mass of fresh mixture restored during the refill process for cases $2.1(1), 2.2(2), 2.3(3)$, and 2.4 (4)

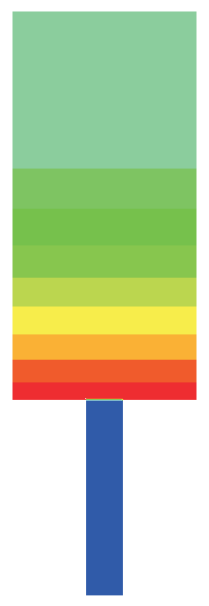

(a)

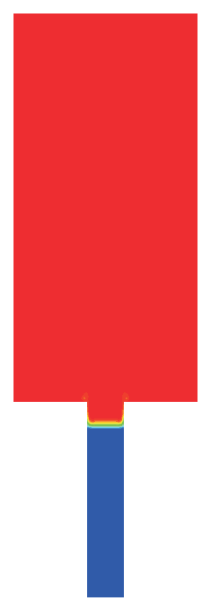

(b)

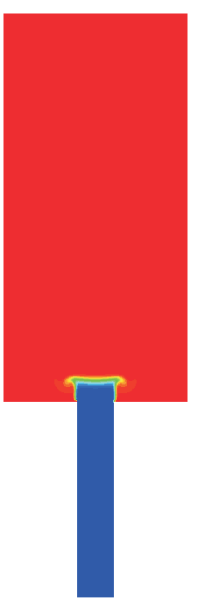

(c)

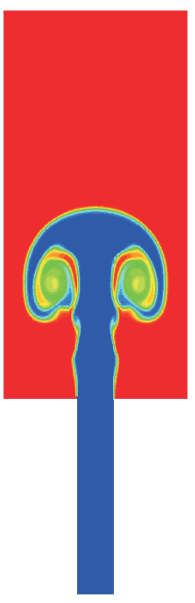

(d)

Figure 12 Two-dimensional results for case 2.2. Initial pressure field $(a)$ and fields of fresh mixture fraction: $(b)$ blocking phase (burnt gases are in red and fresh gases in blue) at $4 \mu \mathrm{s} ;(c)$ refill phase at $10 \mu \mathrm{s}$; and $(d)$ jet development phase at $30 \mu \mathrm{s}$

before the detonation passage. For cases 2.3 and $2.4, \mathrm{M}_{\mathrm{inj}}$ is sonic providing the maximum injection flow rate. Temporal evolutions of $m_{\%}$ are plotted in Fig. 11 for the four cases. When $m_{\%}$ reaches 1 , it means that the initial mass of fresh gases is fully restored in the chamber.

Case 2.2 is illustrated in Fig.12 to show the different injection phases. Figure $12 a$ is the initial pressure field. In Fig. 12b, the burnt gases have still a high pressure leading to a push back effect on the fresh mixture in the feeding 
pipes. Pressure in the chamber continues decreasing and the refill phase begins (Fig. 12c). Finally, the jet develops in the chamber with typical vortex structures shown in Fig. $12 d$.

Cases of separate injection 3.1 to 3.4 are defined in Table 4 similarly to cases 2.1 to 2.4 but for the computational domain shown in Fig. $9 b$. Compared to the previous cases, the refill process is different because the separate injection uses an $\mathrm{H}_{2}$ mass flux $j_{m, \mathrm{H}_{2}}=11.11 \mathrm{~kg} /\left(\mathrm{m}^{2} \mathrm{~s}\right)$ and an $\mathrm{O}_{2}$ mass flux $j_{m, \mathrm{O}_{2}}$ $=88.89 \mathrm{~kg} /\left(\mathrm{m}^{2} \mathrm{~s}\right)$. Both fluxes are related to the chamber cross section given that $A_{\%, \text { inj, } \mathrm{H}_{2}}=1 / 15$ and $A_{\%, \text { inj, } \mathrm{O}_{2}}=2 / 15$. For both propellants, the total injection pressure is now $P_{t, \text { inj }}=0.274 \mathrm{MPa}$ to keep a total mass flux of $100 \mathrm{~kg} /\left(\mathrm{m}^{2} \mathrm{~s}\right)$ with $\mathrm{M}_{\mathrm{ini}}=0.8$. This difference can be seen in Fig. 13 where $m_{\%}$ is plotted vs. time. Contrary to Fig. $11, m_{\%}$ does not reach 1 at $t=50 \mu \mathrm{s}$ whatever the case.

What is more interesting in the case of separate injection is to analyze the equivalence ratio of injected propellants vs. time. In Fig. 13b, one can see that for case 3.2, it takes $50 \mu \mathrm{s}$ to inject the required global ER. It shows the delay that exists between the refill of $\mathrm{H}_{2}$ and $\mathrm{O}_{2}$. The time necessary to obtain the aimed value of global $\mathrm{ER}$ is an important issue in the case of separate injection.

With respect to the CDWRE case, the different phases of the refill process are well reproduced. The time dedicated to the blocking phase represents $20 \%$ of the refill period (defined by the instant when $m_{\%}$ reaches 1 ) and lasts about $10 \mu \mathrm{s}$ for the premix and separate cases with maximum mass flow rates. Compared to

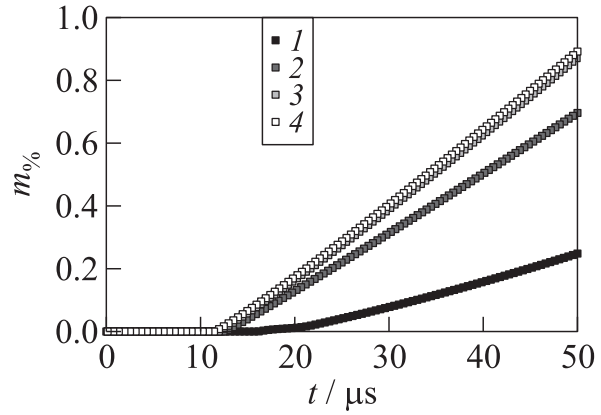

(a)

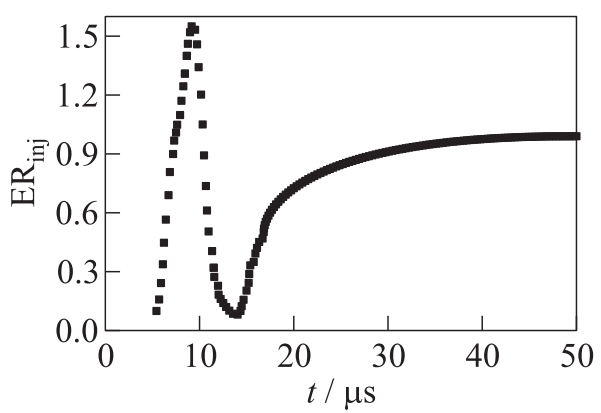

(b)

Figure 13 Refill processes for separate injection cases 3.1 to 3.4 (a) (1 - Case 3.1; 2 - Case 3.2; 3 - Case 3.3; and 4-Case 3.4) and corresponding evolution of global Equivalence Ratio (ER) of injected propellants for case $3.2(b)$ 
the CDWRE case, the process is longer and this will certainly result in a global effect on chamber operation.

\subsubsection{Preparation of three-dimensional unsteady computations}

To adapt the $2 \mathrm{D}$ procedure for the $3 \mathrm{D}$ computations, some modifications have to be done to limit the computational cost. Until now, the feeding pipes were excessively long to prevent the initial shock wave from reflecting at the tube inlets and perturb the injection. Nonreflective boundary conditions have been tested and validated to absorb the shockwave. The tube length can be reduced to 10 diameters. This length is sufficient to compute the burnt gases penetration in the feeding tubes.

Another change consists in unifying the burnt gases composed of six species in one equivalent gas referred to as BG to reduce the number of resolved equations. Let us evaluate the thermodynamic properties of BG: molar mass $W$; enthalpy of formation $h_{f}$; heat capacity at constant pressure $c_{p}$; dynamic viscosity $\mu$; Prandtl and Schmidt numbers. Molar mass $W$ is calculated by harmonic mass weighted average; and $h_{f}$ and $c_{p, i}$ (coefficients of $c_{p}$ ) are calculated by arithmetic molar weighted average. Parameters of the Sutherland law for $\mu_{\mathrm{BG}}$ are obtained with a least squares method to fit the viscosity of the burnt gases evaluated from the Wilke formula. The Prandtl and Schmidt numbers are assumed to be constant for the computations. The Prandtl number is evaluated from the thermal conductivity profile and chosen for a temperature of $2000 \mathrm{~K}$. A constant Schmidt number is evaluated to correspond to a stoichiometric mixture of $\mathrm{H}_{2}$ and $\mathrm{O}_{2}$. These assumptions for the Prandtl and Schmidt numbers might be strong but turbulent transport is supposed to be much stronger than the molecular one in the present simulations.

Finally, to improve results visualization, let us introduce a new variable $Z$, by analogy with the well known mixture fraction, based on molar fractions of the three species $\left(\mathrm{H}_{2}, \mathrm{O}_{2}\right.$, and $\left.\mathrm{BG}\right)$ :

$$
\begin{gathered}
Z_{\mathrm{H}_{2}}=\frac{X_{\mathrm{H}_{2}}}{X_{\mathrm{H}_{2}}+2 X_{\mathrm{O}_{2}}+2 X_{\mathrm{BG}}} ; \\
Z_{\mathrm{O}_{2}}=\frac{2 X_{\mathrm{O}_{2}}}{X_{\mathrm{H}_{2}}+2 X_{\mathrm{O}_{2}}+2 X_{\mathrm{BG}}} \\
Z_{\mathrm{BG}}=\frac{2 X_{\mathrm{BG}}}{X_{\mathrm{H}_{2}}+2 X_{\mathrm{O}_{2}}+2 X_{\mathrm{BG}}}
\end{gathered}
$$

The $Z$ parameter is designed for proper visualization of computational results for the mixing zone. Using mass or mole fractions of the gases is not convenient. Because of very different molar masses, the graphical representation gives the impression that one of the components is dominating. In a stoichiometric mixture without burnt gases, mole fractions of $\mathrm{H}_{2}$ and $\mathrm{O}_{2}$ are related as $X_{\mathrm{H}_{2}} / X_{\mathrm{O}_{2}}$ 
$=2$. From formulas (2) and (3), one obtains for the same mixture $Z_{\mathrm{H}_{2}}=Z_{\mathrm{O}_{2}}$ $=0.5$ that is more convenient for graphical representation of the mixing zone. Now, consider that the stoichiometric mixture is burnt by $50 \%$. By using the global reaction expression $2 \mathrm{H}_{2}+\mathrm{O}_{2}=2 \mathrm{H}_{2} \mathrm{O}$ and substituting $\mathrm{BG}$ for $\mathrm{H}_{2} \mathrm{O}$, one finds $Z_{\mathrm{H}_{2}}=Z_{\mathrm{O}_{2}}=0.25$ and $Z_{\mathrm{BG}}=0.5$ from formula (4). The same values for $Z_{\mathrm{H}_{2}}, Z_{\mathrm{O}_{2}}$, and $Z_{\mathrm{BG}}$ are obtained in the case of dilution by the burnt gases by $40 \%$. Therefore, $Z_{\mathrm{BG}}$ represents a convenient parameter to estimate the degree of dilution or burning of the fresh mixture. We can go further by introducing three more parameters defined by formulas:

$$
\begin{aligned}
Z_{\mathrm{H}_{2}, \text { ex }} & =Z_{\mathrm{H}_{2}}-\min \left(Z_{\mathrm{H}_{2}}, Z_{\mathrm{O}_{2}}\right) ; \\
Z_{\mathrm{O}_{2}, \text { ex }} & =Z_{\mathrm{O}_{2}}-\min \left(Z_{\mathrm{H}_{2}}, Z_{\mathrm{O}_{2}}\right) ; \\
Z_{\mathrm{st}} & =2 \min \left(Z_{\mathrm{H}_{2}}, Z_{\mathrm{O}_{2}}\right)
\end{aligned}
$$

where $Z_{\mathrm{H}_{2} \text {,ex }}$ and $Z_{\mathrm{O}_{2} \text {,ex }}$ represent excess of the respective components from the stoichiometric proportion whereas $Z_{\mathrm{st}}$ is the fraction of stoichiometric mixture. By comparing fields for $Z_{\mathrm{H}_{2} \text {,ex }}, Z_{\mathrm{O}_{2} \text {,ex }}$, and $Z_{\mathrm{st}}$, one can easily differentiate the zones of good and poor mixing. In case of a flow of pure components, the equivalence ratio is a convenient parameter for visualization. However, it is less useful if the mixture quality is affected by dilution. In the latter case, $Z_{\text {st }}$ will help to identify zones where the stoichiometric mixture is nondiluted.

\section{RESULTS FOR THREE-DIMENSIONAL ESTABLISHED INJECTION CASES}

For this computation, the inlet mass fluxes related to the injection sections are the following: $j_{m, \mathrm{O}_{2}}=844 \mathrm{~kg} /\left(\mathrm{m}^{2} \mathrm{~s}\right) ; j_{m, \mathrm{H}_{2}}=213.5 \mathrm{~kg} /\left(\mathrm{m}^{2} \mathrm{~s}\right)$; and $T_{t, \text { inj }}$ $=300 \mathrm{~K}$. The established injection results give a reference flowfield representing what mixing is expected to be after a time long enough for the vortex structures to develop. The Q-criterion is used to visualize the instantaneous eddies in the turbulent flow (as shown in Fig. 14). In Fig. 14a, isosurface of the Q-criterion $\left(10^{8}\right)$ is colored by the value of ER to give an idea about local distribution of ER when the stoichiometric proportions of the propellants are globally respected. The ER color scale is logarithmic from 0.1 to 10 whereas the overall ER variation is from 0 in pure $\mathrm{O}_{2}$ to infinity in pure $\mathrm{H}_{2}$. One can easily deduce from Fig. $14 a$ that the components are not well-mixed in the lower half of the domain, as intermittent zones of unmixed propellants are observed whereas in the upper half, the mixture is almost homogeneous.

This representation does not consider the dilution of the fresh mixture by the burnt gases. As the established results are compared with the unsteady ones, 


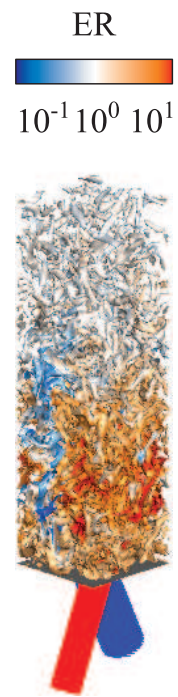

(a)

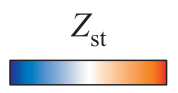

$\begin{array}{lll}0.0 & 0.5 & 1.0\end{array}$
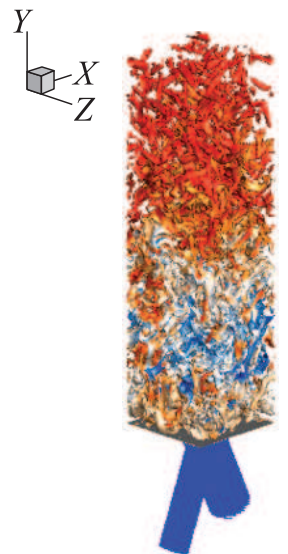

(b)

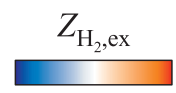

$\begin{array}{lll}0.0 & 0.5 & 1.0\end{array}$

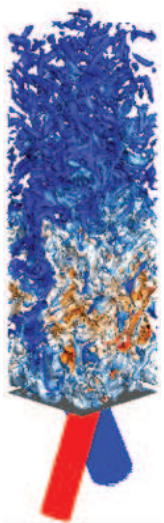

(c)

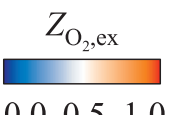

$\begin{array}{lll}0.0 & 0.5 & 1.0\end{array}$

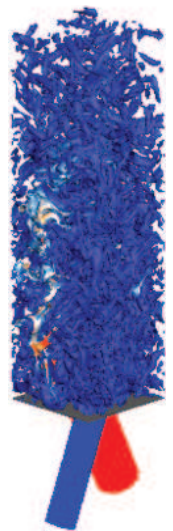

(d)

Figure 14 Instantaneous vortex structures for established injection. Isosurfaces of Q-criterion colored by $\mathrm{ER}(a) ; Z_{\mathrm{st}}(b) ; Z_{\mathrm{H}_{2}, \text { ex }}(c)$; and $Z_{\mathrm{O}_{2}, \text { ex }}(d)$

$Z_{\text {st }}$ is more appropriate as it takes into account the burnt gases fraction; hence, it is possible to distinct the zones filled with well-mixed propellants from the zones with strong dilution by burnt gases.

One can also determine the zones where $\mathrm{H}_{2}$ or $\mathrm{O}_{2}$ are in excess with the parameters $Z_{\mathrm{H}_{2} \text {,ex }}$ or $Z_{\mathrm{O}_{2} \text {,ex }}$. In Fig. 14, it is demonstrated how the different parameters characterize the mixture quality.

Figures $14 a$ and $14 b$ show an equivalent representation of the turbulent mixture because in this established case, there are no burnt gases. Figures $14 \mathrm{c}$ and $14 d$ also show local excess of $\mathrm{H}_{2}$ or $\mathrm{O}_{2}$ close to the injection plane.

From a quantitative point of view, it is worth analyzing the evolution of mixing according to the nondimensional vertical coordinate $y_{a \operatorname{dim}}=y / d_{\mathrm{H}_{2}}$. Instantaneous mixing efficiency can be evaluated from two formulas, with either mass or mass-flow weighting:

$$
\eta_{\mathrm{mix}, \rho}=\frac{\iint_{S_{y}} \rho Y_{\mathrm{H}_{2}} d S / \max (\varphi, 1)}{\iint_{S_{y}} \rho Y_{\mathrm{H}_{2}} d S / \min (\varphi, 1)}
$$




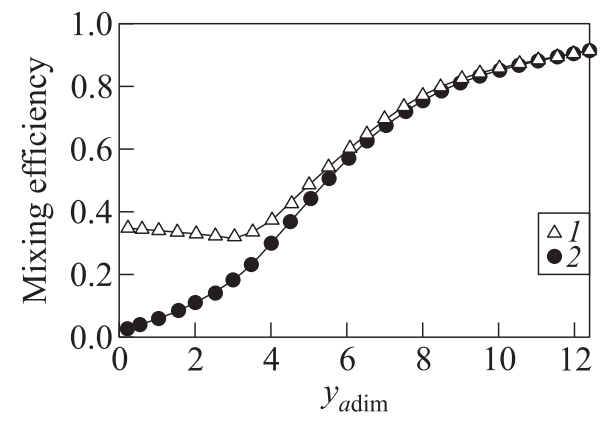

Figure 15 Comparison of time-averaged mixing efficiencies evaluated according to Eqs. (5) (1) and (6) (2) for 3D injection

$$
\eta_{\mathrm{mix}, \dot{m}}=\frac{\iint_{S_{y}} Y_{\mathrm{H}_{2}} d \dot{m} / \max (\varphi, 1)}{\iint_{S_{y}} Y_{\mathrm{H}_{2}} d \dot{m} / \min (\varphi, 1)}
$$

where $\varphi=W_{\mathrm{O}_{2}} Y_{\mathrm{H}_{2}} /\left(2 W_{\mathrm{H}_{2}} Y_{\mathrm{O}_{2}}\right)$ is the local ER, with $W_{\mathrm{O}_{2}}$ and $W_{\mathrm{H}_{2}}$ being the molar mass of $\mathrm{O}_{2}$ and $\mathrm{H}_{2}$, respectively. $\eta_{\text {mix }}$ is varying in the range $[0,1]$, the value of 1 being obtained when $\varphi$ is equal to 1 everywhere in the considered $S_{y}$ section, i. e., when the mixing is perfect in this section.

From the instantaneous profiles, time-averaged efficiencies $\overline{\eta_{\text {mix }, \rho}}$ and $\overline{\eta_{\text {mix }, \dot{m}}}$ are calculated by simple arithmetic average and displayed in Fig. 15. The two efficiencies show similar behavior after $y_{a \mathrm{dim}}=6$. Close to the injection plane, some stagnation and recirculation zones exist. Propellants in these low-velocity zones are partially premixed resulting in $\eta_{\text {mix }, \rho}>0$ at $y=0$.

The mass-flow-weighted efficiency (see Eq. (6)) does not take into account stagnant zones but only the zones of high velocity that are fully unmixed at $y=0$. That is why $\eta_{\mathrm{mix}, \dot{m}}$ is 0 at the beginning and rapidly increases thanks to the jet interactions. Mixing by jet interactions becomes effective from $y_{a \operatorname{dim}}=4$ only where $\eta_{\mathrm{mix}, \rho}$ joins the trend of $\eta_{\mathrm{mix}, \dot{m}}$.

Equation (6) is well adapted to established computations whereas it is not well suited for transitory refill because mass-flow rate is not constant and stabilized.

Equation (5) seems better fitted for transitory refill simulations.

Total pressure losses are not considered in this present study. In [14], it was shown that a strong correlation exists between mixing efficiency and total pressure recovery based on mass-flow weighted average for different injector configurations. 


\section{RESULTS FOR THREE-DIMENSIONAL TRANSITORY INJECTION}

The aim of these simulations is to identify the temporal evolution of the injection and mixing from the instant just after the passage of detonation over the injection element to the achievement of a mixing state comparable with the established situation. For the initial conditions, it was adopted: $\mathrm{M}_{\mathrm{ini}}=0.8$ and $\mathrm{M}_{r h}=1$. The inlets are defined by the following total conditions: $P_{t, \text { inj }}=0.381 \mathrm{MPa}$ and $T_{t, \text { inj }}=300 \mathrm{~K}$. Same mass-weighted mixing efficiency calculations can be done from the instantaneous flowfield. Figure 16 shows instantaneous profiles of mixing efficiency extracted from the transitory computation every $20 \mu \mathrm{s}$. They are compared with the established profile as a reference state. The refill process is supposed to converge toward the stabilized flowfield but at $100 \mu \mathrm{s}$, it has still not completely reached the final state.

The interaction between burnt and fresh gases can be analyzed using parameters $Z_{\mathrm{st}}$ and $Z_{\mathrm{BG}}$. In Fig. 17, upper row, the progression of mixing in the computational domain is illustrated by the isosurfaces of the Q-criterion, colored by $Z_{\text {st }}$, every $20 \mu \mathrm{s}$. The Q-criterion isosurfaces are also shown in Fig. 17, lower row, but colored by $Z_{\mathrm{BG}}$. In the first $20 \mu \mathrm{s}$ (Fig. $17 a$ ), burnt gases are pushed back from the feeding tubes and propellants start to be reinjected in the domain. There are still much burnt gases in the mixing zone (Fig. 17a, lower row) and almost no good mixture (Fig. 17a, upper row).

In the next $20 \mu \mathrm{s}$ (Fig. 17b), the turbulent flow fills the domain and interactions with neighboring injection elements appear as the turbulent structures cross the periodic boundaries. However, mixing is developing in the middle of the domain but is not yet high enough because the flowfield is intermittent.

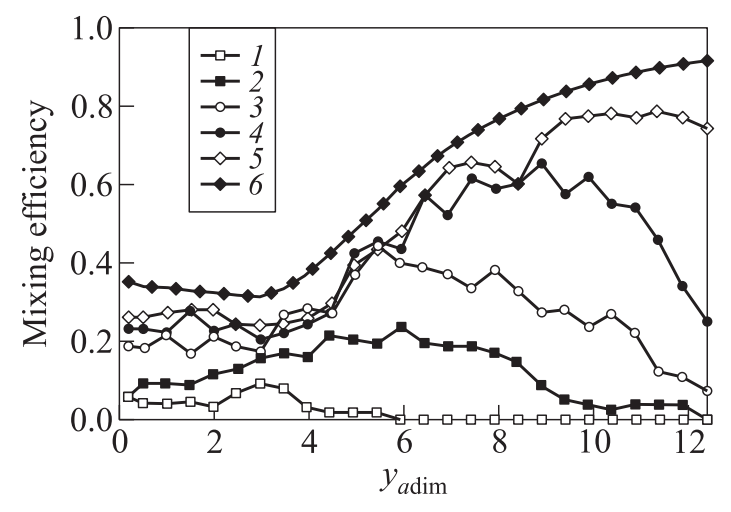

Figure 16 Comparison between instantaneous $(1-20 \mu \mathrm{s} ; 2-40 ; 3-60 ; 4-$ 80 ; and $5-100 \mu \mathrm{s})$ and established $(6) \overline{\eta_{\text {mix }, \rho}}$ profiles 


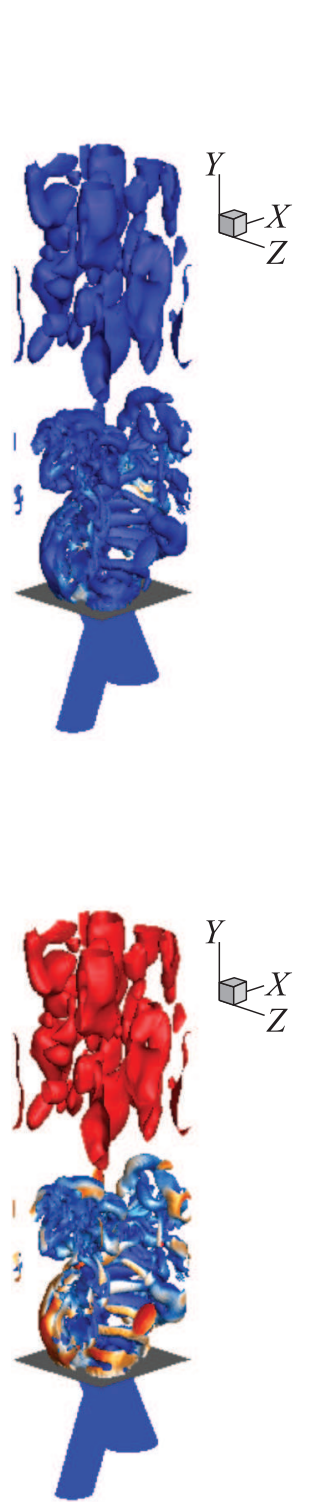

(a)
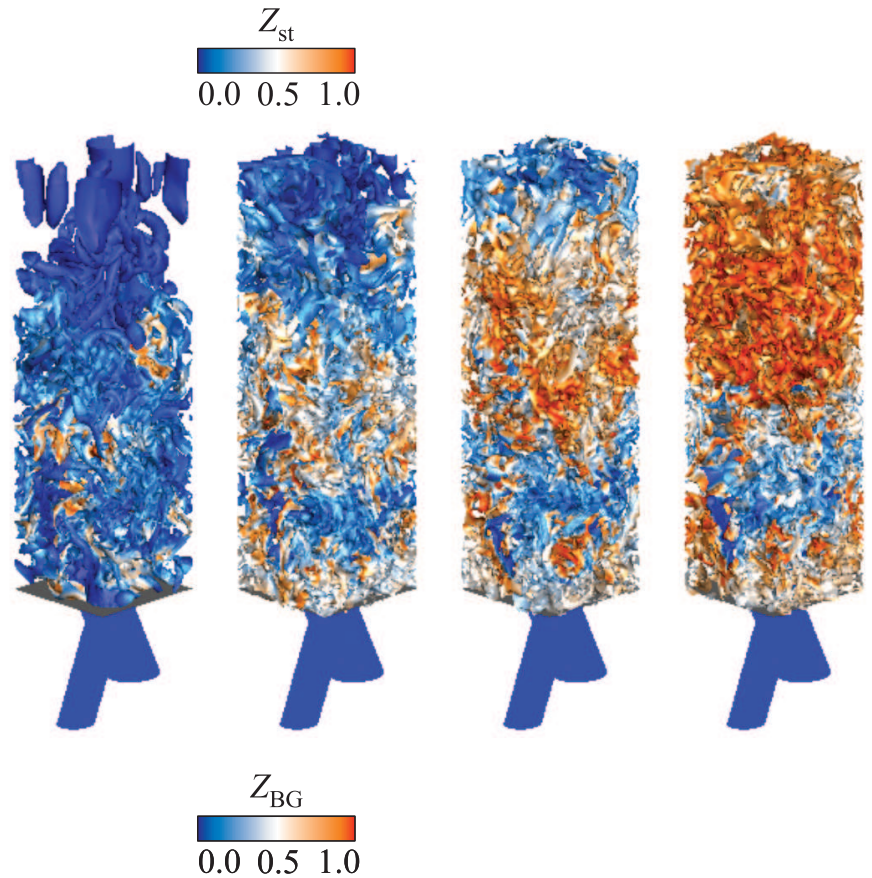

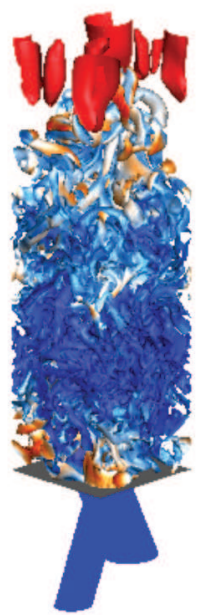

(b)

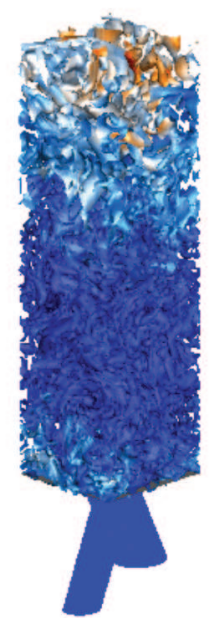

(c)

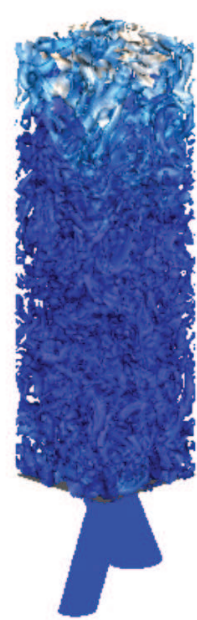

(d)

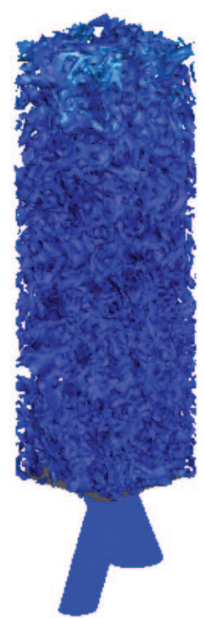

(e)

Figure 17 Instantaneous vortex structures. Isosurfaces of Q-criterion colored by $Z_{\text {st }}$ (upper row) and $Z_{\mathrm{BG}}$ (lower row) for different times of the refill process: (a) $20 \mu \mathrm{s}$; (b) 40; (c) 60; (d) 80; and (e) $100 \mu \mathrm{s}$ 
Some burnt gases remain near the injector face. Between 60 and $100 \mu \mathrm{s}$, turbulent structures become finer and improve the mixing of the propellants. Burnt gases have been rejected from the lower half of the domain but remain in the upper half, as mixing efficiency continues to improve in this part of the domain.

At the end (Fig. 17e), the final mixing state is similar to the one obtained for the established case with a difference from $7 \%$ to $30 \%$, depending on the $y_{a \mathrm{dim}}$ coordinate. This difference seems to be due only to the imperfect mixing as burnt gases have nearly left the domain. The mean $Z_{\mathrm{BG}}$ level in Fig. 17 e (lower row) is $4 \%$.

These results provide an evaluation of the propellant dilution in a nonreacting context. Dilution is related to the mixing of $\mathrm{H}_{2}$ or $\mathrm{O}_{2}$ or both with the surrounding burnt gases and is responsible of two important loss factors. First, a diluted mixture is less energetic; hence, the detonation strength in such a mixture is reduced. Second, diluted mixture can self-ignite before the next detonation passage, resulting in a loss of available mass for detonation. Both factors conduct to a loss of pressure gain in the chamber and by consequence to a loss of RDE performance. With the present 3D methodology, the authors propose the first step in understanding the transient phenomena of propellant injection and mixing occurring between two detonations in a nonreacting environment. A numerical study accounting for chemical reactions and the coupling between the injector response and mixture quality, on the one hand, and the detonation strength and burnt gases expansion, on the other hand, is under way.

\section{CONCLUDING REMARKS}

In this paper, numerical studies were presented in the framework of a global project to simulate CDWRE operation with a realistic injector for separate injection of the propellants. Two numerical procedures were presented. The first procedure is proposed to compute an established injection of the two propellant jets, giving the best mixing state that is expected to be after a long time. The second procedure is aimed at computing the transitory refill phase from the moment the detonation has passed over the injection element. Test cases associated with this procedure have shown that the expansion effect of the burnt gases on the fresh mixture is well reproduced. The results analysis points out that the stabilized conditions are greatly influenced by the Mach number specified for the right state of the Riemann problem. The Mach number in the injection pipe deviates from its initial value during the injection process, thus leading to a mass flow rate change during injection. A 3D transitory simulation was finally performed to study the refill process up to the almost established flow. 


\section{ACKNOWLEDGMENTS}

This study was funded by the General Scientific Directorate of ONERA.

\section{REFERENCES}

1. Zeldovich, Y. 2006. To the question of energy use of detonation combustion. J. Propul. Power 22(3):588-592.

2. Wintenberger, E., and J.E. Shepherd. 2006. Thermodynamic cycle analysis for propagating detonations. J. Propul. Power 22(3):694-698.

3. Zhdan, S. A., A. M. Mardashev, and V.V. Mitrofanov. 1990. Calculation of the flow of spin detonation in an annular chamber. Combust. Explo. Shock Waves 26(2):210-214.

4. Eude, Y., D. M. Davidenko, I. Gokalp, and F. Falempin. 2011. Use of the adaptive mesh refinement for 3D simulations of a CDWRE (continuous detonation wave rocket engine). 17th AIAA Space Planes and Hypersonic Systems and Technologies Conference (International).

5. Schwer, D. A., and K. Kailasanath. 2012. Feedback into mixture plenums in rotating detonation engines. 50th AIAA Aerospace Sciences Meeting including the New Horizons Forum and Aerospace Exposition.

6. Stoddard, W. A., and E. J. Gutmark. 2015. Numerical investigation of centerbodiless RDE design variations. 53rd AIAA Aerospace Sciences Meeting.

7. Kaneshige, M., and E. Shepherd. 1998. Detonation database. GALCIT. Technical Report FM97-8. Available at: http://shepherd.caltech.edu/detn_db/html/db_5.html (accessed June 29, 2017).

8. Andrus, I. Q., P. I. King, M. Fotia, F. R. Schauer, and J. L. Hoke. 2015. Experimental analogue of a pre-mixed rotating detonation engine in plane flow. 53rd AIAA Aerospace Sciences Meeting.

9. Thomas, L. M., F. R. Schauer, J. L. Hoke, and A. Naples. 2011. Buildup and operation of a rotating detonation engine. 49rd AIAA Aerospace Sciences Meeting.

10. Bykovskii, F. A., S. A. Zhdan, and E. F. Verdernikov. 2006. Continuous spin detonations. J. Propul. Power 22(6):1204-1216.

11. Rankin, B.A., D.R. Richardson, A.W. Caswell, A. Naples, J. L. Hoke, and F. R. Schauer. 2015. Imaging of $\mathrm{OH}^{*}$ chemiluminescence in an optically accessible nonpremixed rotating detonation engine. 53rd AIAA Aerospace Sciences Meeting.

12. Stoddard, W. A., and E. J. Gutmark. 2014. Comparative numerical study of RDE injection designs. 52nd AIAA Aerospace Sciences Meeting.

13. Stoddard, W. A., A. St. George, R. Driscoll, and E. J. Gutmark. 2014. Computational analysis of existing and altered rotating detonation engine inlet designs. 50th AIAA/ASME/SAE/ASEE Joint Propulsion Conference. 
14. Gaillard, T., D. Davidenko, and F. Dupoirieux. 2015. Numerical optimisation in non reacting conditions of the injector geometry for a continuous detonation wave rocket engine. Acta Astronaut. 111:334-344.

15. Refloch, A., B. Courbet, A. Murrone, et al. 2011. CEDRE software. AerospaceLab J. Iss. 2. Available at: http://www.aerospacelab-journal.org/CEDRE-Software (accessed June 29, 2017).

16. Scherrer, D., F. Chedevergne, P. Grenard, et al. 2011. Recent CEDRE applications. AerospaceLab J. Iss. 2. Available at: http://www.aerospacelab-journal.org/node/101 (accessed June 29, 2017). 